\title{
Numerical approximation of fluid-structure interaction problems
}

\author{
P. Sváček ${ }^{a, *}$ \\ ${ }^{a}$ Dep. Technical Mathematics, Fac. Mech. Engineering, CTU Prague, Karlovo nám. 13, 12135 Praha 2, Czech Republic \\ Received 8 September 2008; received in revised form 9 October 2008
}

\begin{abstract}
This paper is devoted to numerical approximations of fluid-structure interaction problems. Namely the problem of interaction of turbulent flow over flexibly supported airfoil is addressed. In order to model the turbulence effects the flow is described by Reynolds Averaged Navier-Stokes (RANS) equations. The Reynolds equations and the turbulence model is numerically approximated by the finite element method. In order to avoid spurious oscillations stabilization procedures are applied. The application of the developed numerical method is shown in several numerical experiments.

(c) 2008 University of West Bohemia in Pilsen. All rights reserved.
\end{abstract}

Keywords: finite element method, Arbitrary Lagrangian-Eulerian method, flux corrected transport

\section{Introduction}

The interation of fluid flow and an elastic structure is important in many technical disciplines as aeroelasticity/hydroelasticity, cf. [3]. Frequently linearized models are used in engineering applications. Recently, the problems of nonlinear aeroelasticity began to be important with the arising number of studied situations, see, e.g. [12]. In technical applications the turbulence effects are modelled by Reynolds Averaged Navier-Stokes(RANS) equations together with the Boussinesq approximation of the Reynolds stresses, cf. [15]. The turbulence viscosity is then approximated by a solution of additional partial differential equations (PDE). The approximation of PDEs describing the turbulence model by finite element method is complicated mainly due to the dominating convection. Use of standard SUPG/GLS stabilization methods do not vanish the local undershoots/overshoots effects. To overcome this nonphysical phenomena the additional nonlinear crosswind diffusion can be employed, cf. [2]. Here, we consider the approach based on flux corrected transport (FCT) applied in the finite element context, cf. [9]. The verification of the numerical scheme behaviour is shown in several numerical examples. Further, an aeroelastic model is numericaly approximated.

\section{Mathematical model}

\subsection{Fluid model}

The flow is modelled by Reynolds equations and the Reynolds stresses are approximated by the Boussinesq assumption. The turbulent viscosity is modelled with the aid of $k-\omega$ model, cf. [15]. In order to take into account the deformations of the computational domain, we start with a short introduction of Arbitrary Lagrangian-Eulerian (ALE) method, see also [11, 10, 5].

\footnotetext{
${ }^{*}$ Corresponding author. Tel.: +420 22435 7403, e-mail: Petr.Svacek@ fs.cvut.cz.
} 


\subsubsection{Arbitrary Lagrangian-Eulerian method}

Let us assume that there exists a mapping $\Phi=\Phi(\xi, t)$ defined for any $\xi \in \Omega_{0}$ and $t \in[0, T]$ such that for any $t$ the mapping $\Phi(\cdot, t)$ is a one-to-one transformation of $\Omega_{0}$ onto $\Omega_{t}$. Let us denote $\mathcal{A}_{t}=\Phi(\cdot, t)$. The mapping $\mathcal{A}_{t}$ is called arbitrary Eulerian-Lagrangian mapping (ALE mapping). We assume that for any $t \in I$ the mapping $\mathcal{A}_{t}$ denotes $C^{1}$ continuous bijective mapping from the reference (original) configuration $\Omega_{0}$ onto the domain $\Omega_{t}$ at time $t$ (the current configuration).

The time derivative of the ALE mapping $\mathcal{A}_{t}$ yields the domain velocity $\mathbf{w}_{D}=\mathbf{w}_{D}(x, t)$ for $x \in \Omega_{t}$ and $t \in[0, T]$.

$$
\mathbf{w}_{D}(x, t)=\frac{\partial \Phi}{\partial t}(\xi, t), \quad \mathcal{A}_{t}(\xi)=x, \quad \xi \in \Omega_{0} .
$$

Furthermore, by $D^{\mathcal{A}} / D t$ the $A L E$ derivative is denoted (derivative with respect to a fixed point $\xi$ in the reference domain $\xi \in \Omega_{0}$ ). The ALE derivative is related to the time and spatial derivatives as

$$
\frac{D^{\mathcal{A}} f}{D t}(x, t)=\frac{\partial f}{\partial t}(x, t)+\mathbf{w}_{D}(x, t) \cdot \nabla f(x, t)
$$

for any $x \in \Omega_{t}$ and $t \in(0, T)$.

\subsubsection{Reynolds equations in ALE form}

The Reynolds equations are used written in the ALE form read (cf. [13, 15])

$$
\begin{aligned}
\frac{D^{\mathcal{A}} \mathbf{v}}{D t}-\nabla \cdot\left(\nu_{e f f} \mathbf{S}(\mathbf{v})\right)+(\overline{\mathbf{w}} \cdot \nabla) \mathbf{v}+\nabla p & =0, \\
\operatorname{div} \mathbf{v} & =0, \quad \text { in } \Omega_{t}
\end{aligned}
$$

where $\mathbf{S}(\mathbf{v})=\left(\nabla \mathbf{v}+(\nabla \mathbf{v})^{T}\right), \mathbf{v}$ denotes the vector of mean part of the velocity, $p$ denotes the mean part of the kinematic pressure, $\overline{\mathbf{w}}=\mathbf{v}-\mathbf{w}_{D}, \nu_{\text {eff }}=\left(\nu+\nu_{T}\right), \nu$ denotes the kinematic viscosity, and $\nu_{T}$ denotes the turbulent viscosity.

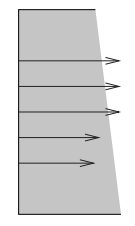

a)

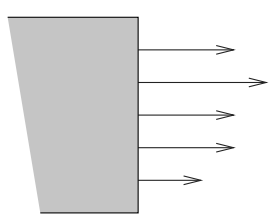

b)

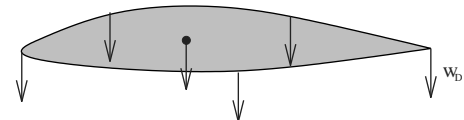

c)

Fig. 1. Different parts of computational domain boundary: a) Dirichlet part of boundary, b) outlet boundary, c) moving airfoil

The system is equipped with the boundary conditions prescribed on the mutually disjoint parts of the boundary $\partial \Omega_{t}$ (see Fig. 1)

$$
\begin{aligned}
& \text { a) } \mathbf{v}(x, t)=\mathbf{v}_{D}(x), \quad x \in \Gamma_{D}, \quad \text { b) } \mathbf{v}(x, t)=\mathbf{w}_{D}(x, t), \quad x \in \Gamma_{W t}, \\
& \text { c) }-\nu_{e f f}\left(\nabla \mathbf{v}+(\nabla \mathbf{v})^{T}\right) \cdot \mathbf{n}+\left(p-p_{\text {ref }}\right) \mathbf{n}=0, \text { on } \Gamma_{O},
\end{aligned}
$$

where $p_{\text {ref }} \in \mathbb{R}, \mathbf{v}_{D} \in \mathbf{H}^{1 / 2}\left(\Gamma_{D}\right), \mathbf{w}_{D} \in \mathbf{H}^{1 / 2}\left(\Gamma_{W t}\right)$ for any $t \in(0, T)$. Moreover, system (3) is equipped with the initial condition $\mathbf{v}(x, 0)=\mathbf{v}_{0}(x)$ for $x \in \Omega_{0}$, where $\mathbf{v}_{0} \in \mathbf{H}^{1}\left(\Omega_{0}\right)$ such that $\nabla \cdot \mathbf{v}_{0}=0$. 


\subsubsection{Turbulence model}

In this paper the turbulence viscosity $\nu_{T}$ is modellad by the k-omega turbulence model (see $[15,8])$ :

$$
\begin{aligned}
\frac{\partial k}{\partial t}+\frac{\partial\left(k v_{j}\right)}{\partial x_{j}} & =P_{k}-\beta^{*} \omega k+\frac{\partial}{\partial x_{j}}\left(\left(\nu+\sigma_{k} \nu_{T}\right) \frac{\partial k}{\partial x_{j}}\right) \\
\frac{\partial \omega}{\partial t}+\frac{\partial\left(\omega v_{j}\right)}{\partial x_{j}} & =P_{\omega}-\beta \omega^{2}+\frac{\partial}{\partial x_{j}}\left(\left(\nu+\sigma_{\omega} \nu_{T}\right) \frac{\partial \omega}{\partial x_{j}}\right)+C_{D}
\end{aligned}
$$

where $\mathbf{v}=\left(v_{1}, v_{2}\right)$ is the velocity vector, $\nu$ is the kinematic viscosity, $k$ is the turbulent kinetic energy, $\omega$ is the specific turbulent dissipation, and $\nu_{T}=k / \omega$ is the kinetic eddy-viscosity coefficient. The production terms are given by

$$
P_{k}=\tau_{i j}^{R} \frac{\partial v_{j}}{\partial x_{i}}, \quad P_{\omega}=\frac{\alpha_{\omega} \omega}{k} P_{k}, \quad C_{D}=\sigma_{D} \frac{1}{\omega} \max \left\{\frac{\partial k}{\partial x_{i}} \frac{\partial \omega}{\partial x_{i}}, 0\right\},
$$

with $\tau^{R}$ the Reynolds-stress tensor. The closure coefficients are chosen according [8], i.e $\beta^{*} / \beta=6 / 5, \alpha_{\omega}=\beta / \beta^{*}-\sigma_{\omega} \kappa^{2} / \sqrt{\beta^{*}}(\kappa=0.41$ is the Von Karman constant $), \beta^{*}=0.09$, $\sigma_{\omega}=0.5, \sigma_{k}=2 / 3$ and $\sigma_{d}=0.5$.

\subsection{Structure model}

The structure is considered to be a flexibly supported airfoil, which can be vertically displaced and rotated. Figure 2 shows the elastic support of the airfoil on translational and rotational springs and its placement in the channel. The governing nonlinear equations are written in the form $($ see $[3,4])$

$$
\begin{array}{r}
m \ddot{h}+S_{\alpha} \ddot{\alpha} \cos \alpha-S_{\alpha} \dot{\alpha}^{2} \sin \alpha+k_{h h} h=-L(t), \\
S_{\alpha} \ddot{h} \cos \alpha+I_{\alpha} \ddot{\alpha}+k_{\alpha \alpha} \alpha=M(t) .
\end{array}
$$

where $m$ is the mass of the airfoil, $S_{\alpha}$ is the static moment of the airfoil around the elastic axis, $I_{\alpha}$ is the inertia moment of the airfoil around the elastic axis, and $k_{h h}$ and $k_{\alpha \alpha}$ are the bending stiffness and torsional stiffness, respectively. The pressure and viscous forces acting on the vibrating airfoil immersed in fluid result in the lift force $L(t)$ and the torsional moment $M(t)$

$$
L=-l \int_{\Gamma_{W t}} \sum_{j=1}^{2} \tau_{2 j} n_{j} d S, \quad M=l \int_{\Gamma_{W t}} \sum_{i, j=1}^{2} \tau_{i j} n_{j} r_{i}^{\text {ort }} \mathrm{d} S,
$$
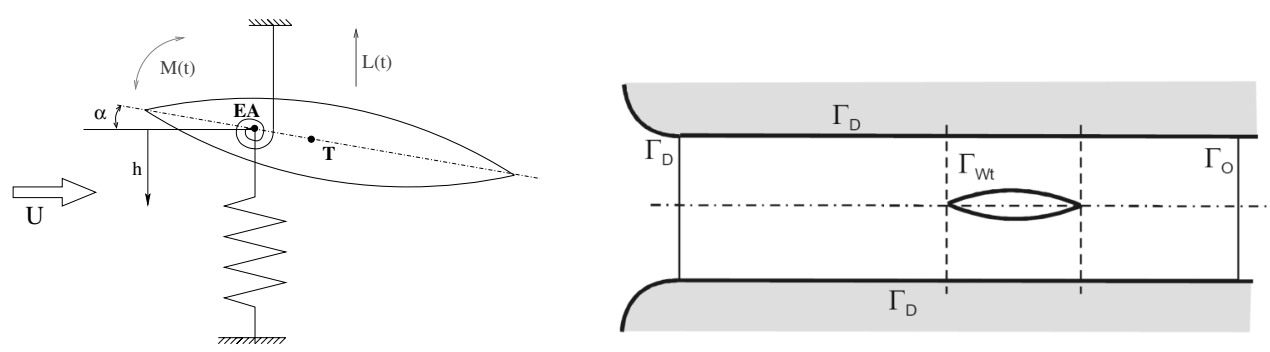

Fig. 2. The elastic support of the airfoil on translational and rotational springs (left) and the computational domain for the channel flow over DCA profile (right) 
where

$$
\tau_{i j}=\rho\left[-p \delta_{i j}+\nu\left(\frac{\partial v_{i}}{\partial x_{j}}+\frac{\partial v_{j}}{\partial x_{i}}\right)\right], \quad r_{1}^{\text {ort }}=-\left(x_{2}-x_{\mathrm{EA} 2}\right), r_{2}^{\text {ort }}=x_{1}-x_{\mathrm{EA} 1}
$$

By $\tau_{i j}$ we denote the components of the fluid stress tensor, $\delta_{i j}$ denotes the Kronecker symbol, $\mathbf{n}=\left(n_{1}, n_{2}\right)$ is the unit outer normal to the domain occupied by surrounding fluid $\Omega_{t}$ on $\Gamma_{W t}$ (pointing into the airfoil) and $x_{\mathrm{EA}}=\left(x_{\mathrm{EA} 1}, x_{\mathrm{EA} 2}\right)$ is the position of the elastic axis (lying in the interior of the airfoil). Relations (8) and (9) define the coupling of the fluid dynamical model with the structural model.

\section{Numerical approximation}

\subsection{Time discretization}

We consider a partition $0=t_{0}<t_{1}<\ldots<T, t_{k}=k \tau$, with a time step $\tau>0$, of the time interval $[0, T]$ and approximate the solution $\mathbf{v}\left(\cdot, t_{n}\right)$ and $p\left(\cdot, t_{n}\right)$ (defined in $\Omega_{t_{n}}$ ) at time $t_{n}$ by $\mathbf{v}^{n}$ and $p^{n}$, respectively. For the time discretization we employ a second-order two-step scheme using the computed approximate solution $\mathbf{v}^{n-1}$ in $\Omega_{t_{n-1}}$ and $\mathbf{v}^{n}$ in $\Omega_{t_{n}}$ for the calculation of $\mathbf{v}^{n+1}$ in the domain $\Omega_{n+1}=\Omega_{t_{n+1}}$.

We define for a fixed time $t=t_{n+1}$ the function spaces for velocity and pressure

$$
\mathcal{W}=\mathbf{H}^{1}\left(\Omega_{n+1}\right), \quad \mathcal{X}=\left\{\mathbf{z} \in \mathcal{W}: \mathbf{z}=0 \text { on } \Gamma_{D} \cup \Gamma_{W t_{n+1}}\right\}, \quad \mathcal{Q}=L^{2}\left(\Omega_{n+1}\right)
$$

We approximate the ALE velocity $\mathbf{w}\left(t_{n+1}\right)$ by $\mathbf{w}^{n+1}$ and set $\widehat{\mathbf{v}}^{i}=\mathbf{v}^{i} \circ \mathcal{A}_{t_{i}} \circ \mathcal{A}_{t_{n+1}}^{-1}$ (the symbol $\circ$ denotes the composite function). The vector-valued functions $\widehat{\mathbf{v}}^{i}$ are defined in the domain $\Omega_{t_{n+1}}$.

Then, on each time level $t_{n+1}$, the second-order two-step ALE time discretization yields the problem of finding unknown functions $\mathbf{v}^{n+1}: \Omega_{t_{n+1}} \rightarrow R^{2}$ and $p^{n+1}: \Omega_{t_{n+1}} \rightarrow R$ satisfying the equations

$$
\begin{aligned}
\frac{3}{2 \tau} \mathbf{v}^{n+1}+\left(\overline{\mathbf{w}}^{n+1} \cdot \nabla\right) \mathbf{v}^{n+1}- & \\
-\nabla \cdot\left(\left(\nu+\nu_{T}\right) \mathbf{S}\left(\mathbf{v}^{n+1}\right)\right)+\nabla p^{n+1} & =\frac{4}{2 \tau} \widehat{\mathbf{v}}^{n}-\frac{1}{2 \tau} \widehat{\mathbf{v}}^{n-1}, \text { in } \Omega_{t_{n+1}} \\
\operatorname{div} \mathbf{v}^{n+1} & =0, \text { in } \Omega_{t_{n+1}}
\end{aligned}
$$

and the boundary conditions (4a-c). Here, $\overline{\mathbf{w}}^{n+1}=\mathbf{v}^{n+1}-\mathbf{w}^{n+1}$. The problem (10) is then weakly formulated in the standard form.

Problem 1 (Weak formulation of Navier-Stokes in ALE form) Find $U=(\mathbf{v}, p)$ such that satisfies

$$
a(U ; U, V)=f(V), \quad \text { for all } V=(\mathbf{z}, q) \in \mathcal{X} \times \mathcal{Q},
$$


and conditions $(4 a, c)$. The forms are defined

$$
\begin{aligned}
a\left(U^{*} ; U, V\right)= & \left(\frac{3 \mathbf{v}}{2 \tau}, \mathbf{z}\right)_{\Omega}+c\left(\overline{\mathbf{w}}^{n+1} ; \mathbf{v}, \mathbf{z}\right)+\int_{\Gamma_{O}} \frac{1}{2}(\mathbf{v} \cdot \mathbf{n})^{+} \mathbf{v} \cdot \mathbf{z} \mathrm{d} S+ \\
+ & \int_{\Omega} \frac{1}{2}\left(\nabla \cdot \mathbf{w}_{D}^{n+1}\right) \mathbf{v} \cdot \mathbf{z} \mathrm{d} x+\left(\left(\nu+\nu_{T}\right) \mathbf{S}(\mathbf{v}), \nabla \mathbf{z}\right)_{\Omega}-(p, \nabla \cdot \mathbf{z})_{\Omega}+(\nabla \cdot \mathbf{v}, q)_{\Omega} \\
f(V)= & \int_{\Omega} \frac{4 \widehat{\mathbf{v}}^{n}-\widehat{\mathbf{v}}^{n-1}}{2 \tau} \cdot \mathbf{z} \mathrm{d} x-\int_{\Gamma_{O}} p_{\mathrm{ref}} \mathbf{z} \cdot \mathbf{n} \mathrm{d} S, \\
& U=(\mathbf{v}, p), V=(\mathbf{z}, q), U^{*}=\left(\mathbf{v}^{*}, p\right),
\end{aligned}
$$

where $\Omega=\Omega_{n+1}, \tilde{\mathbf{w}}=\mathbf{v}^{*}-\mathbf{w}^{n+1}$ and where the trilinear skew-symmetric form $c$ is defined by the relation

$$
c(\mathbf{u} ; \mathbf{v}, \mathbf{w})=\int_{\Omega}\left[\frac{1}{2}(\mathbf{u} \cdot \nabla) \mathbf{v} \cdot \mathbf{w}-\frac{1}{2}(\mathbf{u} \cdot \nabla) \mathbf{w} \cdot \mathbf{v}\right] \mathrm{d} x .
$$

\subsection{Stabilized finite element method}

In order to apply the Galerkin FEM, we approximate the spaces $\mathcal{W}, \mathcal{X}, \mathcal{Q}$ from the weak formulation by finite dimensional subspaces defined over a triangulation $\mathcal{T}_{\Delta}$ of the domain $\Omega_{n+1}$. We use the standard assumptions on the system of triangulation, cf. [1]. Here $\Delta$ denotes the size of the mesh $\mathcal{T}_{\Delta}$. The spaces $W_{\Delta}, \mathcal{X}_{\Delta}$ and $\mathcal{Q}_{\Delta}$ are formed by continuous piecewise linear functions, i.e.

$$
\begin{aligned}
\mathcal{H}_{\Delta}=\left\{v \in C\left(\overline{\Omega_{n+1}}\right) ;\left.v\right|_{K} \in P_{1}(K) \text { for each } K \in \mathcal{T}_{\Delta}\right\} \\
\mathcal{W}_{\Delta}=\left[\mathcal{H}_{\Delta}\right]^{d}, \quad \mathcal{X}_{\Delta}=\mathcal{W}_{\Delta} \cap \mathcal{X}, \\
\mathcal{Q}_{\Delta}=\left\{v \in C\left(\overline{\Omega_{n+1}}\right) ;\left.v\right|_{K} \in P_{1}(K) \text { for each } K \in \mathcal{T}_{\Delta}\right\} .
\end{aligned}
$$

The couple of spaces $\mathcal{X}_{\Delta}$ and $\mathcal{Q}_{\Delta}$ does not satisfy the Babuška-Brezzi inf-sup condition (cf. [14]), so to obtain a stable numerical scheme an additional stabilization needs to be applied, [7]. Moreover, the dominating convection requires further stabilization. Thus we shall use the fully stabilized scheme, cf. ([6]).

We start with the definition of the local element rezidual terms $\mathcal{R}_{K}^{a}, \mathcal{R}_{K}^{f}$ in $K \in \mathcal{T}_{\Delta}$

$$
\mathcal{R}_{K}^{a}(\overline{\mathbf{w}} ; \mathbf{v}, p)=\frac{3 \mathbf{v}}{2 \Delta t}-\nabla \cdot\left(\left(\nu+\nu_{T}\right)\left(\nabla \mathbf{v}+(\nabla \mathbf{v})^{T}\right)\right)+(\overline{\mathbf{w}} \cdot \nabla) \mathbf{v}+\nabla p,
$$

and

$$
\mathcal{R}_{K}^{f}\left(\hat{\mathbf{v}}_{n}, \hat{\mathbf{v}}_{n-1}\right)=\frac{1}{2 \Delta t}\left(4 \hat{\mathbf{v}}_{n}-\hat{\mathbf{v}}_{n-1}\right)
$$

The GLS stabilizing terms are then defined for any $U_{\Delta}=(\mathbf{v}, p) \in \mathcal{X}_{\Delta} \times \mathcal{Q}_{\Delta}, V_{\Delta}=(\mathbf{z}, q) \in$ $\mathcal{X}_{\Delta} \times \mathcal{Q}_{\Delta}$ and $U_{\Delta}^{*}=\left(\mathbf{v}^{*}, p^{*}\right) \in \mathcal{X}_{\Delta} \times \mathcal{Q}_{\Delta}$ :

$$
\begin{array}{r}
\mathcal{L}\left(U_{\Delta}^{*} ; U_{\Delta}, V_{\Delta}\right)=\sum_{K \in T_{\Delta}} \delta_{K}\left(\mathcal{R}_{K}^{a}\left(\overline{\mathbf{w}}^{n+1} ; \mathbf{v}, p\right),\left(\overline{\mathbf{w}}^{n+1} \cdot \nabla\right) \mathbf{z}+\nabla q\right)_{K}, \\
\mathcal{F}\left(U_{\Delta}^{*} ; V_{\Delta}\right)=\sum_{K \in T_{\Delta}} \delta_{K}\left(\mathcal{R}_{K}^{f}\left(\hat{\mathbf{v}}_{n}, \hat{\mathbf{v}}_{n-1}\right),\left(\overline{\mathbf{w}}^{n+1} \cdot \nabla\right) \mathbf{z}+\nabla q\right)_{K},
\end{array}
$$

where the function $\overline{\mathbf{w}}^{n+1}$ stands for the transport velocity, i.e. $\overline{\mathbf{w}}^{n+1}=\mathbf{v}^{*}-\mathbf{w}_{D}^{n+1}$. Further we define

$$
\mathcal{P}_{\Delta}\left(U_{\Delta}, V_{\Delta}\right)=\sum_{K \in \mathcal{T}_{\Delta}} \tau_{K}(\nabla \cdot \mathbf{v}, \nabla \cdot \mathbf{z})_{K}
$$


The following choice of parameters is used

$$
\tau_{K}=\nu_{K}\left(1+R e^{l o c}+\frac{h_{K}^{2}}{\nu_{K} \Delta t}\right), \quad \delta_{K}=\frac{h_{K}^{2}}{\tau_{K}},
$$

where $\nu_{K}=\left|\nu+\nu_{T}\right|_{0,2, K}, h_{K}$ denotes the local element size and the local Reynolds number $R e^{l o c}$ is defined as

$$
R e^{l o c}=\frac{h\|\mathbf{v}\|_{K}}{2 \nu_{K}} .
$$

With the use of the Galerkin terms, GLS terms and grad-div terms the stabilized discrete problem can be formulated in the following way:

Problem 2 (GLS stabilized problem) The stabilized discrete problem: Find $U_{\Delta}=(\mathbf{v}, p) \in$ $\mathcal{W}_{\Delta} \times \mathcal{Q}_{\Delta}$ such that $\mathbf{v}$ satisfies approximately the Dirichlet boundary conditions $(4 a-b)$ and the equation

$$
\begin{array}{r}
a\left(U_{\Delta} ; U_{\Delta}, V_{\Delta}\right)+\mathcal{L}\left(U_{\Delta} ; U_{\Delta}, V_{\Delta}\right)+\mathcal{P}_{\Delta}\left(U_{\Delta}, V_{\Delta}\right) \\
=f\left(V_{\Delta}\right)+\mathcal{F}\left(U_{\Delta}^{*} ; V_{\Delta}\right)
\end{array}
$$

holds for all $V_{\Delta}=(\mathbf{z}, q) \in \mathcal{X}_{\Delta} \times \mathcal{Q}_{\Delta}$.

\section{Flux corrected transport}

The application of the finite element method for approximation of the k-omega turbulence model is complicated due to the several facts: the nonlinearities on the right-hand side, boundary conditions for $\omega$, convection domination. In order to apply the finite element method and to guarantee the positivity of the solution, we shall apply the flux corrected transport (FCT) in the finite element context, [9]. In this paper we focus on description of the main idea of the algorithm. We start with the definition of diffusive and antidiffusive fluxes in the finite element context.

We consider the time dependent continuity equation

$$
\frac{\partial u}{\partial t}+\nabla \cdot(\mathbf{v} u)=0
$$

in a two dimensional domain $\Omega$ with Lipschitz continuous boundary. Here, $u=u(x, t)$ is unknown function defined for any $t \in[0, T]$ and $x \in \Omega, \mathbf{v}$ is a given transport velocity $\mathbf{v} \in$ $\mathrm{C}^{1}(\Omega)$.

In order to approximate equation (20), we assume that the domain $\Omega$ is a polygonal domain triangulated by an addmissible triangulation $\mathcal{T}_{\Delta}$. The functions $u=u(\cdot, t)$ are approximated by piecewise linear continuous functions, i.e.

$$
u(x, t)=\sum_{k} u_{k}(t) \varphi_{k}(x)
$$

where $u_{k}=u_{k}(t)$ are nodal values of the function $u$ at vertices of the triangulation $\mathcal{T}_{\Delta}, \varphi_{k}$ are the standard vertex functions, cf. [1]. This approximation leads to the system of differential equations

$$
M_{C} \underline{\dot{u}}=K \underline{u},
$$


where $\underline{u}=\left(u_{i}\right)$ is vector of the nodal values, $M_{C}=\left(m_{i j}\right)$ is the mass matrix and $K=\left(k_{i j}\right)$ is the discrete transport operator, i.e.

$$
m_{i j}=\int_{\Omega} \varphi_{i}(x) \varphi_{j}(x) \mathrm{d} x, \quad k_{i j}=\int_{\Omega} \nabla \cdot\left(\mathbf{v}(x) \varphi_{i}(x)\right) \varphi_{j}(x) \mathrm{d} x .
$$

The finite element methods produce non-physical undershoots and overshoots in the vincinity of steep gradients. On he other hand, upwind-like approximations are non-oscillatory but overly diffusive. The modern schemes use flux or slope limiters to switch between such linear approximations in an adaptive way. Algebraic constraints are usually applied: it is known that semi-discrete scheme of the form

$$
\frac{d \underline{u}}{d t}=\sum_{j \neq i} c_{i j}\left(u_{j}-u_{i}\right), \quad c_{i j} \geq 0
$$

is local extremum diminishing (LED). The time disretized scheme of equation (22) remains LED if for every solution update from $\underline{u}^{n}$ at time instant $t_{n}$ the new solution $\underline{u}^{n+1}$ satisfies an equivalent algebraic system

$$
A \underline{u}^{n+1}=B \underline{u}^{n},
$$

where $A$ is an M-matrix and $B$ has only non-negative entries.

The above criteria can satisfied with the "discrete upwinding": replace the consistent mass matrix $M_{C}$ by lumped mass matrix $M_{L}$, i.e.

$$
M_{L}=\operatorname{diag} m_{i}, \quad m_{i}=\sum_{j} m_{i j} .
$$

and by adding an artificial diffusion operator $D$ to operator $K$ to eliminate all negative offdiagonal coefficients of $K$, for instance we choose $D$ such that

$$
d_{j i}=d_{i j}=\max \left(-k_{i j}, 0,-k_{j i}\right), \text { for } i \neq j, d_{i i}=-\sum_{j \neq i} d_{i j}
$$

The linear LED scheme then reads

$$
M_{L} \underline{\dot{u}}=L \underline{u}, \quad L=K+D .
$$

The artificial diffusion operator $D$ is symmetric matrix with zero row and column sums, so that can be rewritten as

$$
(D \underline{u})_{i}=-\sum_{j \neq i} f_{i j}^{d}, f_{i j}^{d}=d_{i j}\left(u_{i}-u_{j}\right)=-f_{j i}^{d},
$$

where $f_{i j}^{d}$ are the diffusive fluxes. The original scheme can be written in this context as

$$
M_{L} \underline{\dot{u}}=L \underline{u}-D \underline{u}+\left(M_{L}-M_{C}\right) \underline{u},
$$

or componet by component

$$
m_{i} \dot{u}_{i}=\sum_{j} l_{i j} U_{j}+\sum_{j \neq i} f_{i j}, \quad f_{i j}=f_{i j}^{d}+m_{i j}\left(\dot{u}_{i}-\dot{u}_{j}\right)=-f_{j i},
$$

where $m_{i}$ are coefficients of the lumped mass matrix $M_{L}=\operatorname{diag}\left(m_{i}\right), m_{i}=\sum_{j} m_{i j}$. In order to prevent the oscillations of the solution, the fluxes $f_{i j}$ are multiplied by suitable correction factors

$$
f_{i j}^{*}=\alpha_{i j} f_{i j}, \text { where } 0 \leq \alpha_{i j} \leq 1 .
$$

Inserting these fluxes into (23) we get the nonlinear combination of the low order scheme $\left(\alpha_{i j}=0\right)$ and the original higher order scheme $\left(\alpha_{i j}=1\right)$. More detailed description can be found in [9]. 


\section{Numerical results. Conclusion}

\subsection{Flux corrected transport tests}

First, the developed flux corrected transport scheme was tested for finite element implementation in 1d (see Figs. 3-4) for constant transport velocity, i.e. numerical solution of equation

$$
u_{t}+u_{x}=0
$$

was computed with $\Delta t=10^{-3}$ and $T=0.5$. The exact solution in this case is transported from the left to the right. The previously described procedure was applied on this case on matrices arising from the finite element discretization on equidistant mesh with $\Delta x=10^{-2}$. Four different initial conditions were prescribed. The numerical solutions are shown in Figs. 3-4: by the dashed line the initial condition is shown, the dotted line denotes the exact solution at $T=0.5$ and by the solid line the numerical approximation by the finite element method together with FCT limiting is shown. In all cases, the solution is in agreement with other method and shows that the method is well applicable for the transport problems.
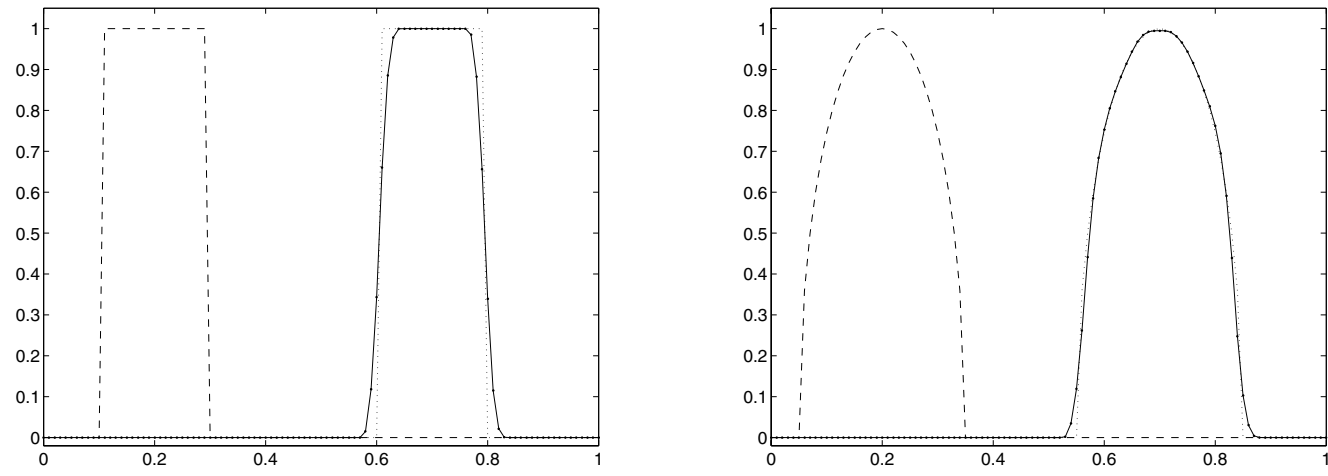

Fig. 3. The numerical solution of transport problem by FCT scheme: Convection of rectangle (left) and semiellipse (right). Dashed line - initial condition, dotted - exact solution, solid line - numerical approximation
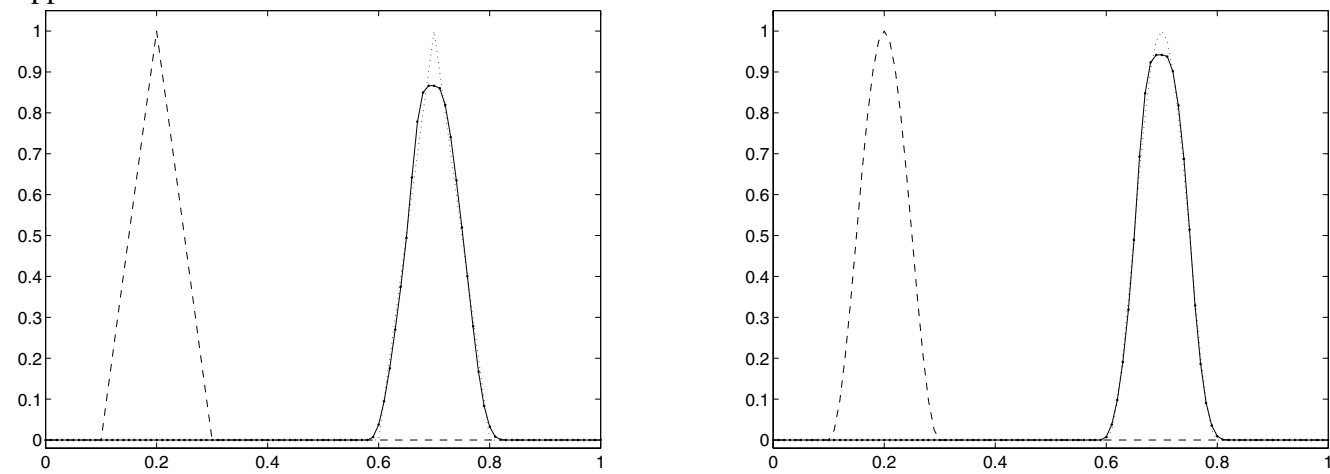

Fig. 4. The numerical solution of transport problem by FCT scheme: Convection of triangle (left) and parabolla (right). Dashed line - initial condition, dotted - exact solution, solid line - numerical approximation 

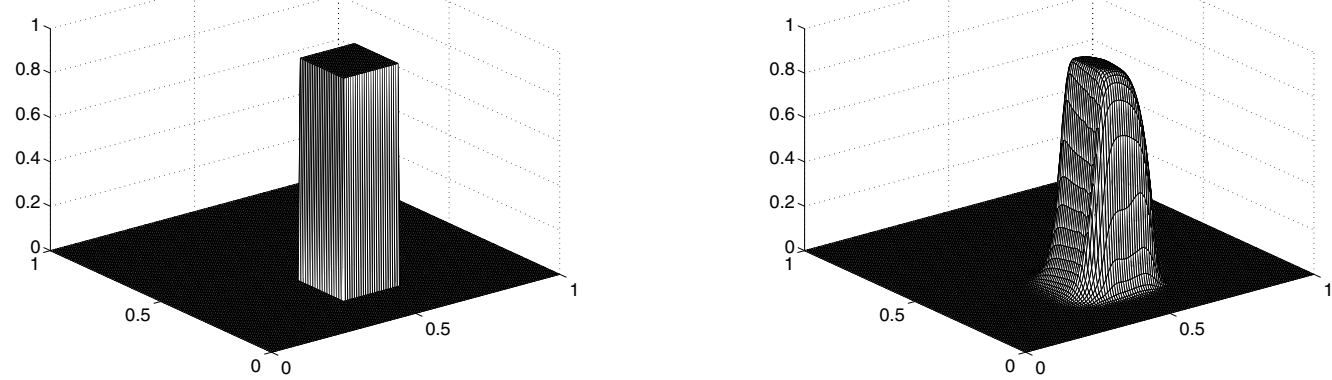

Fig. 5. The $2 \mathrm{~d}$ numerical solution of transport problem by FCT scheme: the exact solution and the initial approximation (left) and numerical solution(right)

Further, a problem in $2 \mathrm{~d}$ was solved: Find $u: \Omega \mapsto \mathbb{R}$ such that

$$
u_{t}+\nabla \cdot(\mathbf{v} u)=0,
$$

where $\mathbf{v}(x, y)=(0.5-y, 0.5-x)$ is divergence free velocity. The exact solution is constant on each circle trajectory with the center at point $[0.5,0.5]$ and periodic in time with the period $T=2 \pi$. Thus the exact solution after one period $2 \pi$ is the same as the initial condition. Numerical result of this test problem is shown in Fig. 5 for the inital condition $u_{0}(x, y)=1$ for $x \in[0.4,0.6]$ and $y \in[0.2,0.4]$. In the numerical solution a spreading the jump, is observed due to added artificial diffusion, but the numerical solution still does not have any undershoots or overshoots.

\subsection{Aeroelastic simulations}

The numerical results of aeroelastic simulations are shown for the case studied in [4]. The following values of structural parameters were used

$$
\begin{aligned}
& m=0.086622 \mathrm{~kg}, \quad S_{\alpha}=-0.000779673 \mathrm{~kg} \cdot \mathrm{m}, \quad I_{\alpha}=0.000487291 \mathrm{~kg} \cdot \mathrm{m}^{2}, \\
& k_{h h}=105.109 \mathrm{~N} \cdot \mathrm{m}^{-1}, \quad k_{\alpha \alpha}=3.695582 \mathrm{~N} \cdot \mathrm{m} \cdot \mathrm{rad}^{-1}, \quad l=0.05 \mathrm{~m}, \quad c=0.3 \mathrm{~m} .
\end{aligned}
$$

The elastic axis is located at $40 \%$ of the airfoil, $\rho=1.225 \mathrm{~kg} \cdot \mathrm{m}^{-3}, \nu=1.5 \cdot 10^{-5} \mathrm{~m} \cdot \mathrm{s}^{-2}$. The numerical computations were performed for airfoil NACA 0012. The aeroelastic response in $h$ and $\alpha$ is shown in Figs. 6-11. The numerical simulation agrees with the linear Theodorsen theory, which predicts the critical velocity $U_{\infty}=37.7 \mathrm{~m} / \mathrm{s}$. For velocities lower than $37 \mathrm{~m} / \mathrm{s}$ the aeroelastic does not predict any instability. For velocity $45 \mathrm{~m} / \mathrm{s}$ the typical flutter type of instability can be observed.

\subsection{Conclusion}

In this paper a numerical method for approximation of aeroelastic problems was described. It is based on application of stabilized finite element method on Reynolds Averaged Navier-Stokes equations, solution of system of ordinary differential equations for structure motion and coupling algorithm. Moreover, the turbulence is modelle by two equation $k-\omega$ turbulence model. In order to guarantee the monotonicity of approximation of $k, \omega$, the idea of flux corrected transport. The numerical solution of several test cases is shown. Further, the developed numerical scheme was applied on an aeroelastic problem. The critical speed is correctly predicted and post-flutter behaviour is shown. 

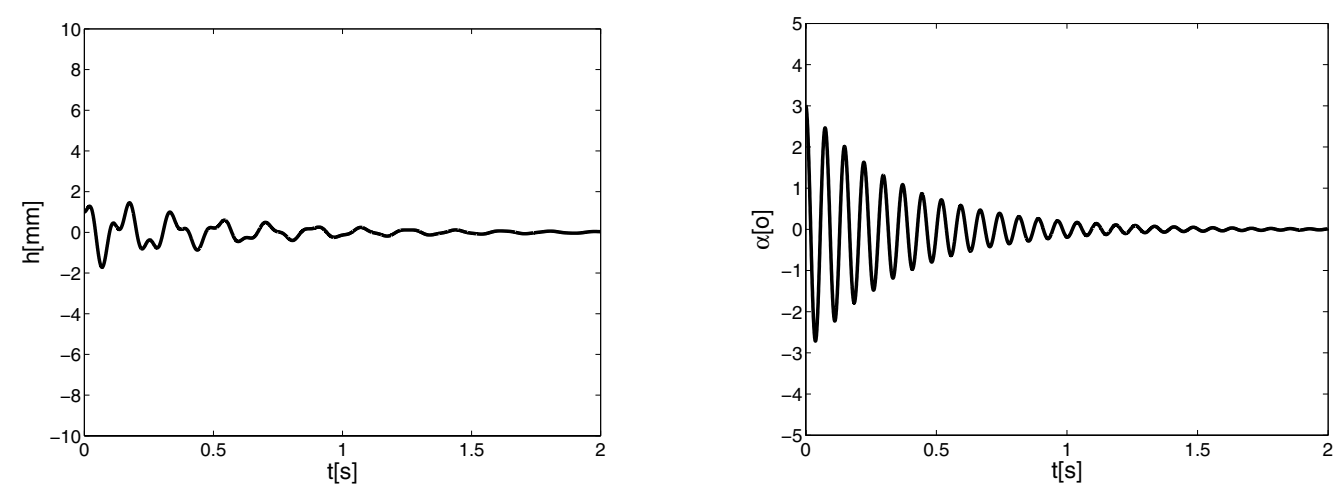

Fig. 6. Aeroelastic response for far field velocity $U_{\infty}=10 \mathrm{~m} / \mathrm{s}$
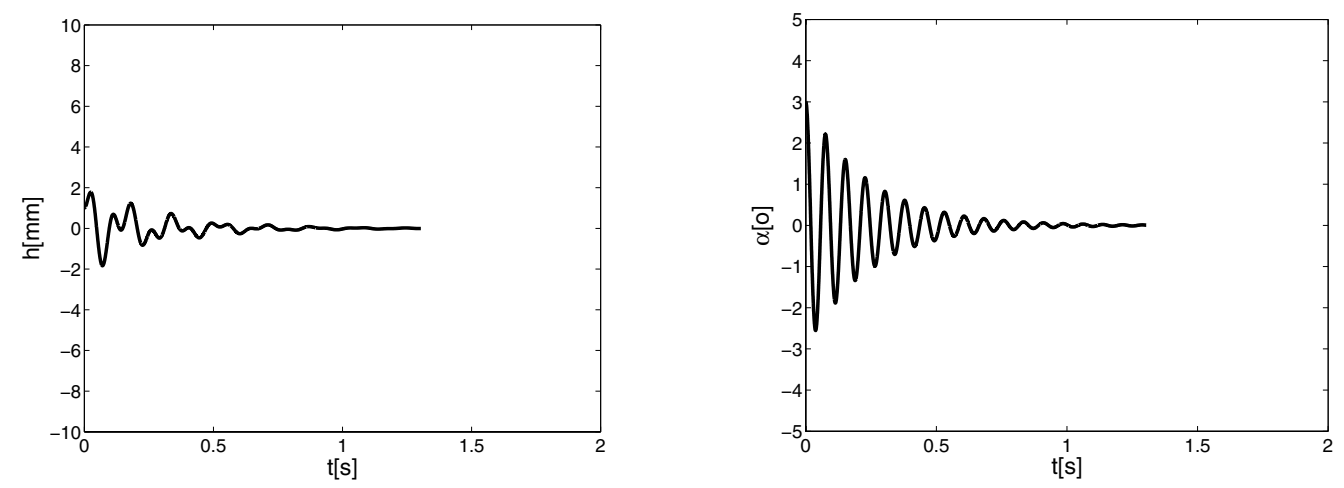

Fig. 7. Aeroelastic response for far field velocity $U_{\infty}=20 \mathrm{~m} / \mathrm{s}$
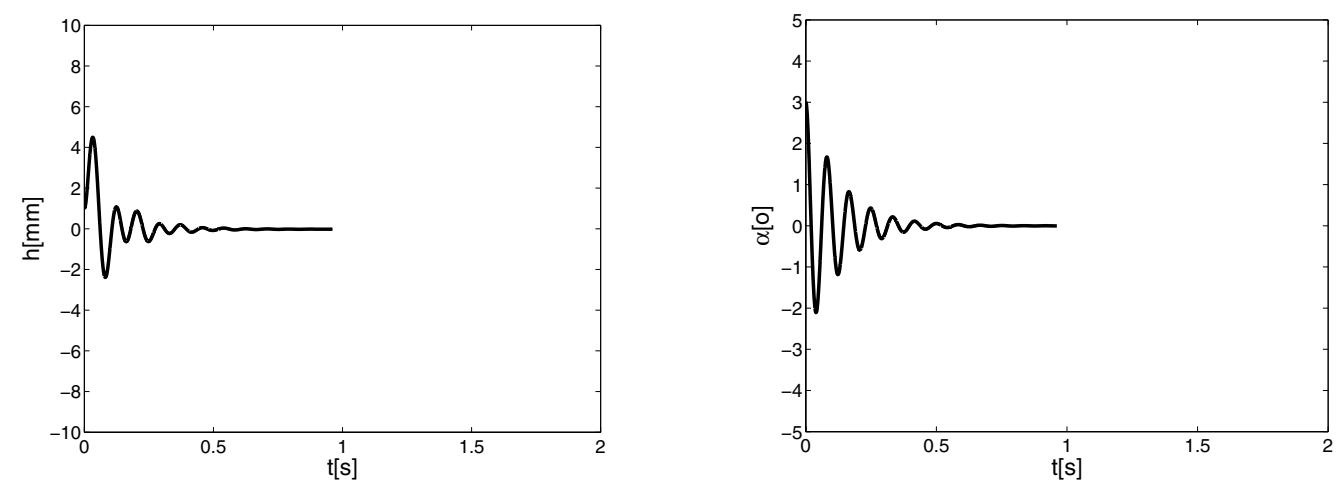

Fig. 8. Aeroelastic response for far field velocity $U_{\infty}=25 \mathrm{~m} / \mathrm{s}$ 

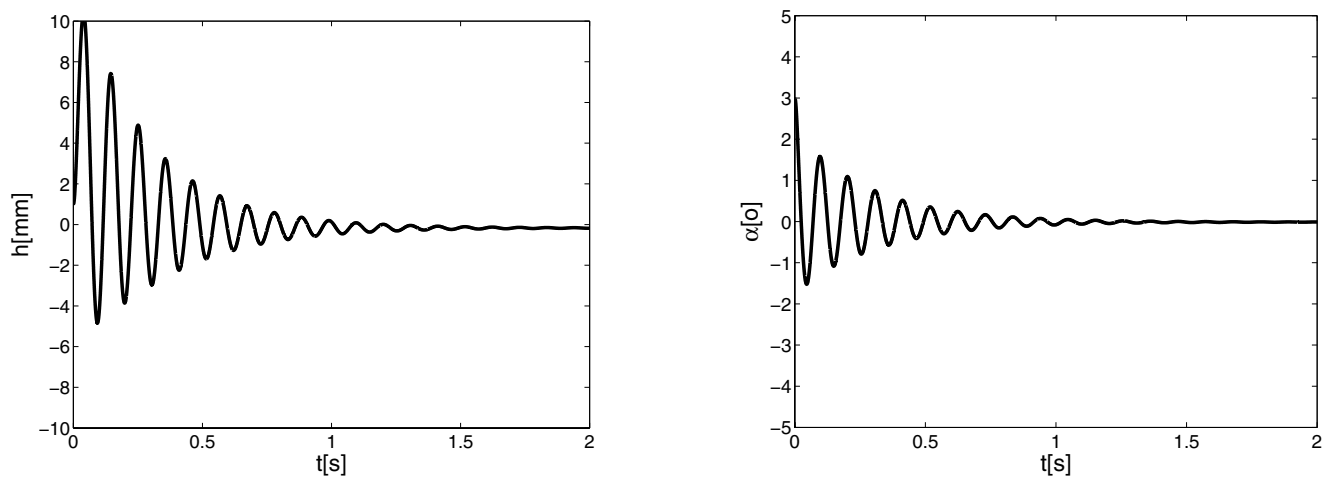

Fig. 9. Aeroelastic response for far field velocity $U_{\infty}=35 \mathrm{~m} / \mathrm{s}$
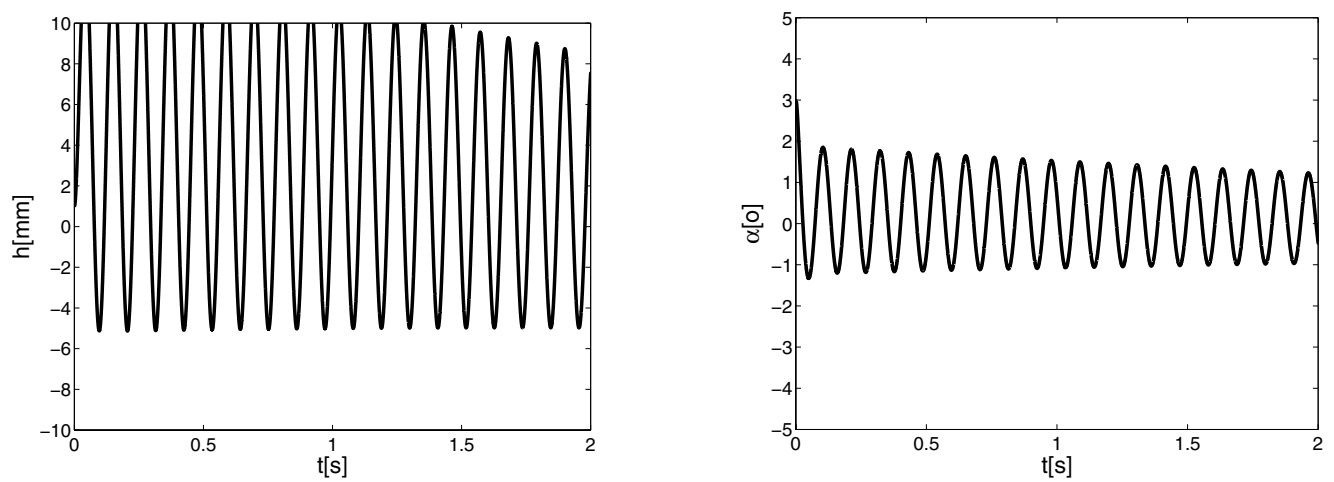

Fig. 10. Aeroelastic response for far field velocity $U_{\infty}=38 \mathrm{~m} / \mathrm{s}$
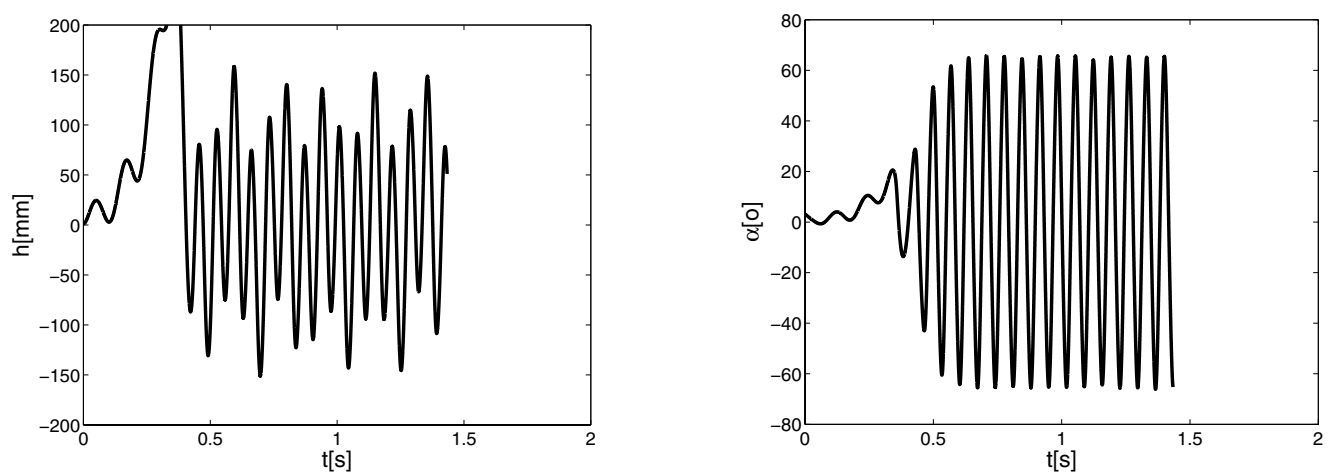

Fig. 11. Aeroelastic response for far field velocity $U_{\infty}=45 \mathrm{~m} / \mathrm{s}$ 


\section{Acknowledgements}

This research was supported under grant No. 201/08/0012 of the Grant Agency of the Czech Republic and under the Research Plan MSM 6840770003 of the Ministry of Education of the Czech Republic.

\section{References}

[1] P. G. Ciarlet, The Finite Element Methods for Elliptic Problems, North-Holland Publishing, 1979.

[2] R. Codina, A discontinuity capturing crosswind-dissipation for the finite element solution of the convection diffusion equation. Computational Methods in Applied Mechanical Engineering, 110:325-342, 1993.

[3] E. H. Dowell, A Modern Course in Aeroelasticity, Kluwer Academic Publishers, Dodrecht, 1995.

[4] M. Feistauer, J. Horáček, P. Sváček, Numerical simulation of flow induced airfoil vibrations with large amplitudes. Journal of Fluids and Structure, 23(3):391-411, 2007. ISSN 0889-9746.

[5] Ch. Förster, W. A. Wall, E. Ramm, On the geometric conservation law in transient flow calculations on deforming domains. International Journal For Numerical Methods In Fluids, 50:1 369-1 379, 2006.

[6] T. Gelhard, G. Lube, M. A. Olshanskii, J.-H. Starcke, Stabilized finite element schemes with LBBstable elements for incompressible flows. Journal of Computational and Applied Mathematics, 177:243-267, 2005.

[7] T. J. R. Hughes, L. P. Franca, M. Balestra, A new finite element formulation for computational fluid dynamics: V. Circumventing the Babuška-Breezzi condition: a stable Petrov-Galerkin formulation of the Stokes problem accomodating equal order interpolation. Computer Methods in Applied Mechanical Engineering, 59:85-89, 1986.

[8] J. C. Kok, Resolving the dependence on free-stream values for the k-omega turbulence model, Technical report, National Aerospace Laboratory NLR, 1999.

[9] D. Kuzmin. On the design of general-purpose flux limiters for finite element schemes. i. scalar convection, Journal of Computational Physics, 219:513-531, 2006.

[10] F. Nobile, Numerical approximation of fluid-structure interaction problems with application to haemodynamics, Ph.D. thesis, Ecole Polytechnique Federale de Lausanne, 2001.

[11] T. Nomura, T. J. R. Hughes, An arbitrary Lagrangian-Eulerian finite element method for interaction of fluid and a rigid body. Computer Methods in Applied Mechanics and Engineering, 95:115-138, 1992.

[12] E. Onate, M. Papadrakakis, B. Schrefler, editors, International Center for Numerical Methods in Engineering (CIMNE), Barcelona, Spain, 2007.

[13] S. B. Pope, Turbulent Flows, Cambridge, University Press, 2000.

[14] R. Verfürth, Error estimates for mixed finite element approximation of the Stokes equations, R.A.I.R.O. Analyse numérique/Numerical analysis, 18:175-182, 1984.

[15] D. C. Wilcox, Turbulence Modeling for CFD, DCW Industries, 1993. 